\title{
Structural Characteristics of Human Substantia Nigra Neuromelanin and Synthetic Dopamine Melanins
}

\author{
K. L. Double, *L. Zecca, *P. Costi, †M. Mauer, †C. Griesinger, $¥$ S. Ito, §D. Ben-Shachar, \\ $\|$ G. Bringmann, IIR. G. Fariello, **P. Riederer, and **M. Gerlach \\ Prince of Wales Medical Research Institute, Sydney, New South Wales, Australia; *Institute of Advanced Biomedical \\ Technologies-CNR, Segrate, Italy; †Institute for Organic Chemistry, Johann Wolfgang Goethe University, \\ Frankfurt, Germany; $\$$ School of Health Sciences, Fujita Health University, Toyoake, Aichi, Japan; \\ $\S$ Laboratory of Psychobiology, Department of Psychiatry, Rambam Medical Centre, B. Rappaport, Faculty of Medicine, \\ Technion, Haifa, Israel; $\mathbb{I}$ Department of Neurology, T. Jefferson University, Philadelphia, Pennsylvania, U.S.A.; and \\ **Clinical Neurochemistry, Department of Psychiatry and ||Department of Organic Chemistry, \\ Julius-Maximilians University of Würzburg, Würzburg, Germany
}

\begin{abstract}
Neuromelanin (NM) is a complex polymer pigment found primarily in the dopaminergic neurons of the human substantia nigra. The structure of NM is only partially characterized, and its synthesis pathway remains unknown. We used nuclear magnetic and infrared spectroscopy to examine the structure of human NM isolated from the substantia nigra compared with synthetic dopamine melanins. Biochemical analyses were used to investigate proteinaceous and dopaminergic components in these samples. Following acid hydrolysis of NM samples, small amounts of DOPA, dopamine, and a variety of amino acids were measured. These findings suggest a peptide component in NM structure. NM also appears to contain a variety of unidentified structural components possibly derived from the oxidation of dopamine. Human NM differs structurally from synthetic dopamine melanin, but both human and synthetic NM include an aromatic backbone. It is interesting that both human NM and synthetic melanin also contain a large proportion of aliphatic structures. Our results suggest that NM is a more complex pigment than synthetic dopamine melanin formed via dopamine autoxidation alone. Key Words: Neuromelanin-Iron-Protein-Nuclear magnetic resonance-Dopamine neurons-Parkinson's disease. J. Neurochem. 75, 2583-2589 (2000).
\end{abstract}

Neuromelanin (NM) is a partially characterized pigment found primarily in catecholaminergic neurons of the human substantia nigra (SN) and locus coeruleus (LC). Little is known of NM's structure or synthesis, although the association of NM with the primary catecholaminergic systems of the brain implicated these substances in the biosynthesis of NM. The two major forms of NM are generally regarded as the result of the spontaneous autoxidation of dopamine (DA) and noradrenaline in the SN and LC, respectively, although an enzymatic synthesis pathway is not to be excluded. The resulting macropolymer appears to be composed of the aminochromes dopaminochrome (in the $\mathrm{SN}$ ) and noradrenalinochrome (in the LC) (Smythies, 1996). SN NM, which has been studied more intensively than that of the LC, was proposed to be a copolymer of the two main classes of melanin: the black insoluble eumelanins composed of indole monomers and the brown alkali-soluble pheomelanin produced from oxidized cysteinyl-DOPA products (Odh et al., 1994). The dopaminergic metabolite 5-S-cysteinyldopamine (cysDA), derived from the $o$-quinone intermediate by the addition of L-cysteine or glutathione and uncyclized DA, may also be present (Rosengren et al., 1985; Carstam et al., 1991; Zhang and Dryhurst, 1994). Other work suggests that NM does not include cysDA (Wakamatsu et al., 1991). A significant amount of sulfur is present, probably as heterocyclic compounds such as benzothiazines (Zecca et al., 1992; Zecca and Swartz, 1993). Solid-state nuclear magnetic resonance (NMR) suggests the presence of a glycidic and lipid matrix and perhaps aliphatic alcoholic groups from the hydroxylation of melanin (Aime et al., 1996). Other constituents remain to be identified and are likely to include inorganic components, particularly iron (Zecca et al., 1992), and possibly a proteinaceous component (Gerlach et al., 1995). A complete elemental analysis of NM was reported by Zecca et al. (1992), indicating the formula $\mathrm{C}_{24.3} \mathrm{H}_{34.2} \mathrm{~N}_{2.7} \mathrm{O}_{10.7} \mathrm{~S}_{1}$.

Received March 13, 2000; revised manuscript received June 30, 2000; accepted July 14, 2000.

Address correspondence and reprint requests to Dr. K. L. Double at Prince of Wales Medical Research Institute, Barker St., Randwick, Sydney, NSW 2031, Australia. E-mail: K.Double@unsw.edu.au

Drs. K. L. Double and L. Zecca contributed equally to this work.

Abbreviations used: cysDA, cysteinyldopamine; cysDAM, dopamine melanin prepared with cysteine; DA, dopamine; DAM, dopamine melanin; LC, locus coeruleus; NM, neuromelanin; NMR, nuclear magnetic resonance; PD, Parkinson's disease; SN, substantia nigra. 
No physiological function has been established for $\mathrm{NM}$, but it has been implicated in the pathogenesis of Parkinson's disease (PD). NM-containing cells of the SN are reported to be more vulnerable to neurodegeneration in PD (Hirsch et al., 1988), and patients suffering a drug-induced parkinsonian syndrome during life exhibited changes in NM consistent with an active disease process (Langston et al., 1999). Increased iron levels are reported in the parkinsonian SN (Ben-Shachar and Youdim, 1991), and iron bound to NM granules has been directly identified (Good et al., 1992; Jellinger et al., 1992; Zecca and Swartz, 1993). In the presence of iron, melanin can act as an effective prooxidant in vitro (Pilas et al., 1988; Zareba et al., 1995), a mechanism suggested to result in the intraneuronal production of free radicals in vivo. The increased free radical level is proposed to be a mechanism initiating neurodegeneration, especially given the compromised state of antioxidative mechanisms in this disorder (see, for example, Youdim et al., 1989; Ben-Shachar and Youdim, 1990).

In contrast, Gibb (1992) has suggested that NM may confer an advantage to the cells in which it is found via its ability to sequester a variety of potentially damaging substances, such as iron (Zecca and Swartz, 1993; Gerlach et al., 1994; Zecca et al., 1994, 1996). Synthetic melanins also exhibit radical-scavenging properties (Sarna et al., 1985, 1986; Zareba et al., 1995) and both synthetic and human NM inhibit iron-induced lipid peroxidation in vitro (Ben-Shachar et al., 1991; Double et al., 1999). A range of potentially toxic dopaminergic metabolic products have recently been identified (Bindoli et al., 1992; Zhang and Dryhurst, 1994; Li and Dryhurst, 1997; Shen et al., 1997; Shen and Dryhurst, 1998), leading to the suggestion that the conversion of such products to NM may represent a cellular detoxification process (Smythies, 1996). These contrasting data epitomize the enigmatic nature of NM and highlight the need to understand its physiological role. Further, knowledge regarding the structure of NM will be the critical first step in clarifying its synthetic pathway and perhaps providing clues for its function and possible role in disease. We have chosen techniques that exploit the known properties of human SN NM to investigate its structural properties in comparison with those of synthetic DA melanin (DAM).

\section{EXPERIMENTAL PROCEDURES}

\section{Isolation of human NM}

NM was isolated according to the method of Zecca and Swartz (1993) from the SN of 18 human subjects from Germany (age range: $29-81$ years) with no history of neurological or neurodegenerative diseases. The $\mathrm{SN}$ were dissected from the brain on a cool plate $\left(-10^{\circ} \mathrm{C}\right)$ within $36 \mathrm{~h}$ of death and pooled in a glass-Teflon homogenizer. The samples were homogenized in $20 \mathrm{ml}$ water and centrifuged at $12,000 \mathrm{~g}$ for $10 \mathrm{~min}$. The resulting pellets were washed twice with $50 \mathrm{~m} M$ phosphate buffer ( $\mathrm{pH} 7.4$ ), then incubated in $50 \mathrm{~m} M$ Tris buffer ( $\mathrm{pH} 7.4)$ containing $0.5 \mathrm{mg} / \mathrm{ml}$ sodium dodecyl sulfate at $37^{\circ} \mathrm{C}$ for $3 \mathrm{~h}$, followed by a further 3 -h incubation with or without the addi- tion of $0.2 \mathrm{mg} / \mathrm{ml}$ proteinase $\mathrm{K}$ in the same buffer. The resulting pellets were pooled and washed consecutively with saline, water, methanol, and hexane. The resulting dark pellet was incubated for three periods of $8 \mathrm{~h}$ each in $150 \mathrm{~m} M$ EDTA ( $\mathrm{pH}$ 7.4) before being washed twice with water and finally dried under vacuum. Further samples were isolated from subjects in Italy using the same method.

\section{Preparation of synthetic NMs}

Synthetic DAM was prepared according to a previously described method (Ben-Shachar et al., 1991). DA (2 mM; Sigma, U.S.A.) was incubated in $50 \mathrm{mM}$ Tris buffer ( $\mathrm{pH} \mathrm{8)}$ containing $0.1 \mathrm{mM} \mathrm{CuSO}_{4}$ for $24 \mathrm{~h}$ at $26^{\circ} \mathrm{C}$. Oxidation was stopped by the addition of concentrated $\mathrm{HCl}$ to $\mathrm{pH} 2$, and the resulting liquid was centrifuged at $9,000 \mathrm{~g}$ for $15 \mathrm{~min}$. The pellet was resuspended three times in $0.01 \% \mathrm{KCl}$ and centrifuged at 27,000 $\mathrm{g}$ for $20 \mathrm{~min}$; the final pellet was resuspended by homogenization in $0.01 \% \mathrm{KCl}$ and dialyzed in doubledistilled water at $4^{\circ} \mathrm{C}$ for $48 \mathrm{~h}$. The resulting melanin was finally lyophilized. A further sample of synthetic melanin was prepared with the addition of cysteine (cysDAM) at a molar ratio of 6:1 DA:cysteine as previously described (Zecca et al., 1994).

\section{Spectroscopic analysis}

Infrared spectra were obtained with a Perkin-Elmer Model 1600 FTIR spectrophotometer using a potassium bromide pellet. NMR spectra were recorded using a Bruker DRX600 (600 $\mathrm{MHz})$ and a Bruker DRX800 (800 MHz) spectrometer. Samples of NM or DAM were dissolved in $0.2 M \mathrm{NaOD}(99.5 \%$; Deutero $\mathrm{GmbH}$, Germany) or deuterated dimethyl sulfoxide $\left(\mathrm{Me}_{2} \mathrm{SO}-\mathrm{d}_{6}\right)$ (99.9\%; Deutero $\left.\mathrm{GmbH}\right)(\sim 80 \%$ solubilization of the polymer was achieved), and the supernatant was measured at $298 \mathrm{~K}$; data were transformed using Bruker XWin NMR software.

\section{Biochemical analyses}

The presence of amino acids in NM was determined using two different methods. Amino acids were analyzed in the Italian samples by hydrolysis of $\sim 0.2 \mathrm{mg}$ of $\mathrm{NM}$ in $0.4 \mathrm{ml}$ of $6 \mathrm{M} \mathrm{HCl}$ in an evacuated sealed tube at $110^{\circ} \mathrm{C}$ for $24 \mathrm{~h}$. After evaporation, amino acids were analyzed by a Hitachi amino acid analyzer. Threonine and serine were assumed to be decomposed by 5 and $10 \%$ under the hydrolysis conditions: the reported values were compensated accordingly for this decomposition. Cysteine was analyzed after hydrolysis with hydroiodic acid (HI) after the method of Ito et al. (1988). Amino acid analysis of NM isolated from a German population was performed using a similar method (Gieseg et al., 1993) to that used by the Italian laboratory (L.Z.). In brief, $0.2 \mathrm{mg}$ of the NM sample was weighed into a small bowl and placed in a desiccator on the porcelain shelf. After the addition of $2 \mathrm{ml}$ of $6 \mathrm{M}$ $\mathrm{HCl}$ containing $1 \%$ phenol and $100 \mu \mathrm{l}$ of thioglycol as antioxidants in the bottom of the desiccator, it was gassed with argon and evacuated using a water aspirator. Finally, the desiccator was placed in a dry oven for $16 \mathrm{~h}$ at $110^{\circ} \mathrm{C}$. The hydrolyzed samples were dissolved in $100 \mu \mathrm{l}$ of $0.3 \mathrm{M} \mathrm{HCl}$, and amino acids were detected by reverse-phase HPLC with fluorescence detection, using a previously published method (Gerlach et al., 1996). Additionally, DOPA and 5-cysteinylDOPA were measured by HPLC with electrochemical detection. DA and DOPA were also assessed simultaneously in both the Italian and German samples in the Japanese laboratory using HI hydrolysis as previously reported (Ito and Fujita, 1985; Ito et al., 1988). 


\section{RESULTS}

NMR spectroscopy demonstrated that human NM isolated in the presence or absence of proteinase K, DAM, and cysDAM exhibited common peaks in the aromatic region at $8.4,7.05,6.85,6.5$, and $6.4 \mathrm{ppm}$, and a peak at $7.3 \mathrm{ppm}$ was also usually observed in most spectra (Fig. 1). Additionally, the signal of the aromatic protons (6-9 $\mathrm{ppm}$ ) exhibited splitting due to $3 \mathrm{~J}$ couplings between vicinal protons (Fig. 2). In two-dimensional NMR correlation (COSY) spectra (data not shown), cross-peaks between the signals at 6.4 and $6.85 \mathrm{ppm}$, and 7.3 and 7.4 ppm, respectively, were observed.

The aliphatic region of both NM and cysDAM exhibited higher spectral activity than the aromatic region; this was most apparent in NM (Fig. 1). The spectra of both $\mathrm{NM}$ and cysDAM show peaks corresponding to methylene and methine groups (2-4 ppm). The signals at $\sim 1$ ppm might be attributed to methyl groups of the aliphatic component in human NM. This cannot be the case, however, for the synthetic DAM and cysDAM. The signals here might arise from methylene protons located close to the center of an aromatic ring system where they experience a high-field shift. This hypothesis is supported by the finding of stacking of the aromatic backbone in NM (Crippa et al., 1996).

The suggestion that protein is intrinsic to the structure of $\mathrm{NM}$ is supported by a comparison of the infrared spectra from NM and DAM. Spectra obtained with human NM exhibited additional peaks; that at $3,000 \mathrm{~cm}^{-1}$ could be attributed to the presence of aliphatic groups, in agreement with previous work on infrared spectroscopy of NM (Bridelli et al., 1999). Further, additional peaks in the NM spectra were apparent between 900 and 1,300 $\mathrm{cm}^{-1}$ and may be due to double carbon bonds and carbon-nitrogen bonds, respectively (Fig. 3).

An analysis of the amino acid content following $\mathrm{HCl}$ hydrolysis of human NM isolated from Italian brains that had been used previously for the NMR spectra studies indicated varying quantities of amino acids. The amino acid glycine was most abundant, followed by serine and proline, whereas the least abundant amino acids were valine, cysteine, and tyrosine (Table 1). Analysis of the amino acid content of NM isolated from German brains using gas-phase $\mathrm{HCl}$ hydrolysis found similar results (Table 1). Samples of DAM and isolated human NM were also analyzed for amino acid content in Japan; the total amino acid content of human NM was dependent on proteinase treatment, whereas no amino acids were detected in DAM (Table 2).

It is interesting that, following HI hydrolysis of human NM, small amounts of DOPA and DA were measured (Table 2). DAM, in contrast, generated a large amount of DA, but, as expected, DOPA was not detected. Using the same method, 5-cysteinyl-DOPA was not detected in $\mathrm{NM}$, with or without proteinase $\mathrm{K}$ treatment, but was present in a small amount $(2.4 \mu \mathrm{g} / \mathrm{mg})$ in DAM.

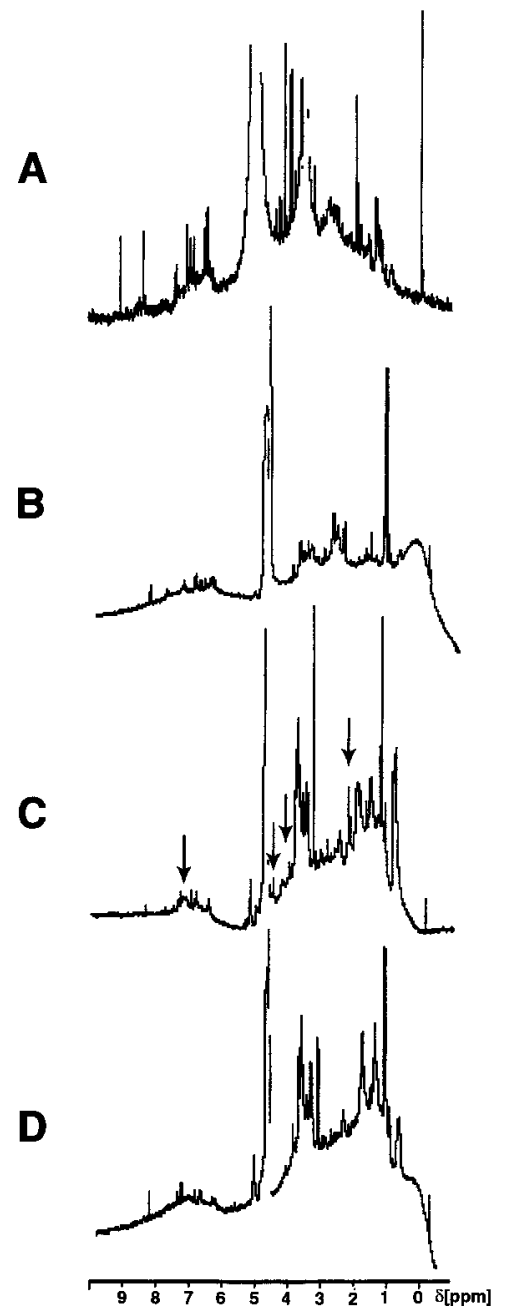

FIG. 1. NMR spectra obtained for DAM, cysDAM, and isolated human SN NM dissolved in 0.2 M NaOD. A: Spectrum obtained from DAM prepared without cysteine. B: Spectrum obtained for cysDAM prepared with cysteine, exhibiting its characteristic form. C: Spectrum obtained from human SN NM isolated in the absence of proteinase. Four additional peaks not present in NM following proteinase treatment are indicated with arrows, supporting the suggestion that NM contains a proteinaceous component. D: Spectrum obtained from human SN NM isolated in the presence of proteinase exhibited additional peaks not seen in those from synthetic melanin. The number of peaks in the aliphatic region indicates that NM consists primarily of an aliphatic chain structure and a smaller backbone of aromatic structures. The aliphatic structures are associated with the melanin polymer itself, rather than with the attached proteinaceous components, as they are also characteristic of DAM and human NM following proteinase treatment.

\section{DISCUSSION}

Spectra of NM samples obtained using proton NMR of solubilized NM exhibited a characteristic pattern. Peaks between 6.4 and $8 \mathrm{ppm}$ are characteristic for aromatic protons, and those between 0 and $5.2 \mathrm{ppm}$ for aliphatic protons, with the aliphatic region being more prominent for both the synthetic and human NM (Fig. 1). The 


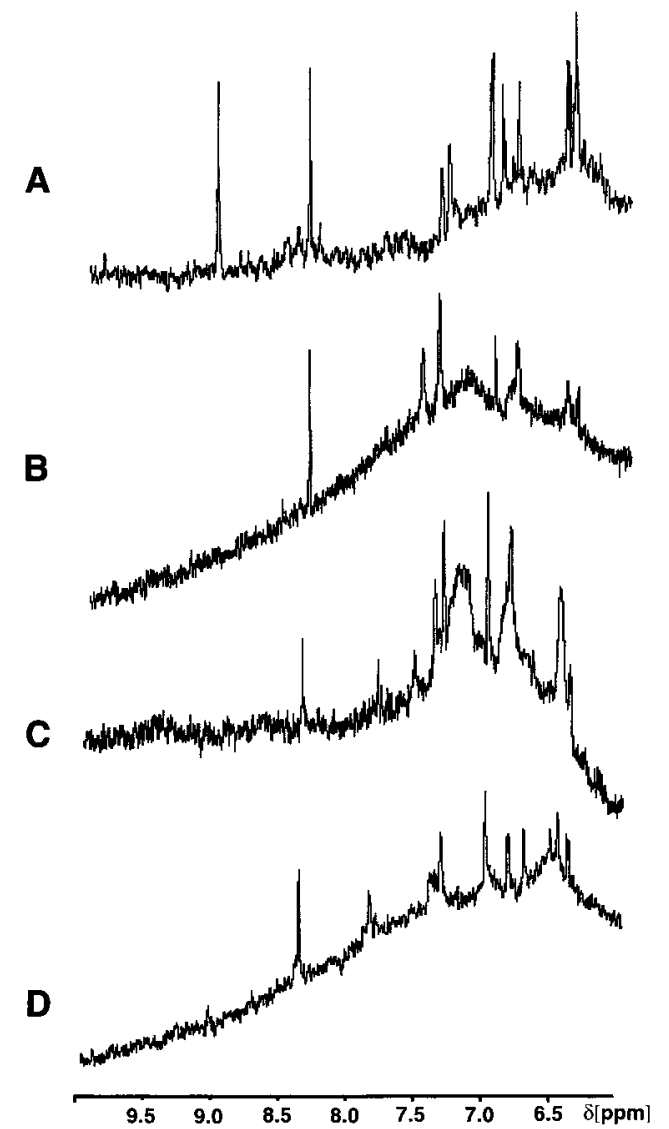

FIG. 2. NMR spectra from (A) DAM, (B) cysDAM, (C) NM without protease treatment, and (D) NM after protease treatment showing the spectral region of signals from aromatic protons. All samples were dissolved in $0.2 \mathrm{M}$ NaOD. DAM, cysDAM, and human NM exhibit common peaks in the aromatic region at 8.4 , $7.6,6.8,6.6$, and $6.4 \mathrm{ppm}$, which may reflect fundamental structural characteristics describing the aromatic backbone of both synthetic melanin and human NM. The signal pattern of cysDAM resembles human NM more closer than that of DAM.

aliphatic structures are likely to be associated with the melanin polymer itself, rather than with attached proteinaceous components, as they are also seen in both melanin types when the amino acid content is minimal following proteinase treatment. This concords with data of an elemental analysis previously reported (Bridelli et al., 1999) showing that the $\mathrm{H} / \mathrm{C}$ ratio in $\mathrm{NM}$ is higher than the $\mathrm{H} / \mathrm{C}$ ratio in synthetic melanin. A fundamental structural characteristic of melanin appears to be the aromatic backbone seen in cysDAM, DAM, and human NM. This finding is in agreement with a recent report using solid-state NMR that identified a signal from human SN melanin that corresponds to a signal produced by aromatic carbons (Lopiano et al., 1999). Further structural information is provided by the observed splitting in the aromatic proton signals that arise from $3 \mathrm{~J}$ couplings of vicinal protons as indicated by two-dimensional correlation spectra (data not shown). Given the strongly alkaline conditions under which the spectra were measured ( $\mathrm{pH} \mathrm{13)}$, it is unlikely that this splitting is attributable to nitrogen-bound pro- tons. Proposals regarding the structure of the soluble component of NM must, therefore, satisfy the requirement of aromatic ring systems with at least two protons in vicinal positions. The NMR data do not exclude the possibility that these aromatic systems belong to a mixture of compounds, rather than to a single molecule. Signals at $\sim 7.3-7.4 \mathrm{ppm}$ might thus come from the aromatic ring system of DA, whereas signals at 6.9 and $6.4 \mathrm{ppm}$ might be attributed to vicinal protons in the heterocyclic ring of dihydroxyindole formed by sidechain cyclization of DA.

Several additional peaks apparent in the spectra obtained from human NM were absent in the synthetic melanin and may be due to peptides bound to the polymer. Comparison of spectra from NM isolated with or without proteinase $\mathrm{K}$ also suggest that these peaks reflect a protein component (Fig. 1B and D). The loss of four peaks at $\sim 7.3 \mathrm{ppm}$ and between 0 and $5 \mathrm{ppm}$ following proteinase treatment suggests that these signals represent protein moieties linked to the NM polymer. The protein content appears to be minor, however, as most of the aliphatic signals are retained following proteinase treatment. The measured signal width was small, which in-
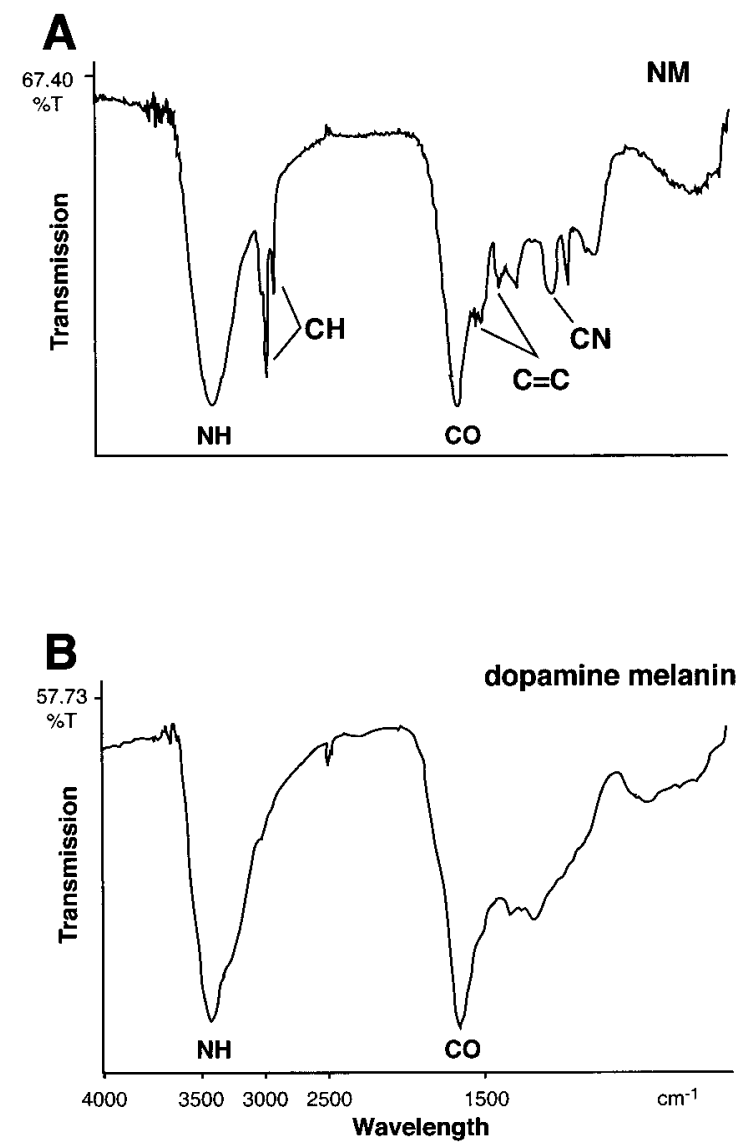

FIG. 3. Infrared spectra obtained from human NM and DAM. The additional peak apparent at $3,000 \mathrm{~cm}^{-1}$ may be attributed to the presence of aliphatic groups, whereas the additional peaks apparent between 900 and $1,300 \mathrm{~cm}^{-1}$ for NM may be due to double carbon bonds and carbon-nitrogen bonds, respectively. 
TABLE 1. Amino acid content of NM

\begin{tabular}{lcc}
\hline Amino acid & $\begin{array}{c}\text { NM A } \\
(\mathrm{nmol} / \mathrm{mg})\end{array}$ & $\begin{array}{c}\text { NM B } \\
(\mathrm{nmol} / \mathrm{mg})\end{array}$ \\
\hline Aspartic acid & $62.7 \pm 23.1$ & 27.4 \\
Threonine & $41.9 \pm 17.9$ & 12.3 \\
Serine & $85.0 \pm 28.1$ & 19.1 \\
Glutamic acid & $70.7 \pm 23.1$ & 34.4 \\
Proline & $50.3 \pm 2.2$ & $\mathrm{n} . \mathrm{m}$. \\
Glycine & $109.2 \pm 36.0$ & 33.6 \\
Alanine & $53.5 \pm 15.5$ & 24.5 \\
Valine & $31.9 \pm 19.8$ & 16.9 \\
Cysteine & $10.6 \pm 3.7$ & $\mathrm{n} . \mathrm{m}$. \\
Isoleucine & $58.9 \pm 19.4$ & $\mathrm{n} . \mathrm{m}$. \\
Leucine & 37.12 & $\mathrm{n} . \mathrm{m}$. \\
Tyrosine & $17.9 \pm 6.1$ & 8.3 \\
Phenylalanine & $41.6 \pm 11.4$ & $\mathrm{n} . \mathrm{m}$. \\
Lysine & $66.8 \pm 20.2$ & $\mathrm{n} . \mathrm{m}$. \\
Histidine & $27.4 \pm 12.9$ & 9.7 \\
Methionine & $\mathrm{n} . \mathrm{m}$. & 5.3 \\
Arginine & $44.3 \pm 12.5$ & n.m. \\
\hline
\end{tabular}

NM A was isolated from Italian brains, and data represent means $\pm \mathrm{SD}$ of three determinations with the exception of leucine, which was measured once. NM B was isolated from German brains, and a single analysis of amino acids was performed. Isolation and $\mathrm{HCl}$ hydrolysis procedures were similar for both samples. n.m., not measured.

dicates a relatively small molecular weight (between 1,000 and 5,000) in the solubilized NM, but the underlying broad background under the sharp signals suggests the presence of a paramagnetic signal as previously reported (Zecca et al., 1996). Spectra obtained from NM and DAM dissolved in $\mathrm{Me}_{2} \mathrm{SO}-\mathrm{d}_{6}$ also show a number of sharp signals in the aromatic and aliphatic regions, although in $\mathrm{Me}_{2} \mathrm{SO}-\mathrm{d}_{6}$ more peaks in the aromatic region were observed. Due to the different conditions in the two solvents $\mathrm{Me}_{2} \mathrm{SO}-\mathrm{d}_{6}$ and NaOD, the spectra are not comparable and therefore are not shown here. The variety of signals observed thus appears not to be attributable to artefactual production of melanin fragments by the alkaline conditions.

A comparison of the coupling patterns of DAM and cysDAM with NM (Fig. 2A and B) suggest that NM shares some features of both spectra, although cysDAM appears to model human NM more closely than DAM. Both NM and cysDAM share a similar aromatic backbone structure that may consist of DA oxidation products, such as dopachrome. The differences between the spectra from NM and cysDAM, however, indicate that the synthetic product differs from NM, and is thus unlikely to model human NM adequately. This finding concords with that of Aime et al. (1996), who showed, using solid-state NMR, that NM has a "peculiar and characteristic structure compared with other melanins from both natural and synthetic origin."

The cyclization of DA-quinone is much slower than that of dopaquinone (Peter and Förster, 1989); thus, uncyclized aliphatic side chains of DA and the presence of amino acids may contribute to the aliphatic region of the spectra. Our NMR results support the findings of Zecca et al. (1992), who reported the presence of long- chain alkyl compounds in human NM and suggest that human NM consists of indole rings bound to aliphatic moieties. The indole rings may be derived from DA oxidation as has been reported (Rosengren et al., 1985; Zhang and Dryhurst, 1994), although this is not found by all groups (Wakamatsu et al., 1991). Recently, it has been demonstrated that cysteinyl adducts of L-DOPA, DA, and 3,4-dihydroxyphenylacetic acid can be demonstrated in the human brain postmortem and that increased levels of these products exist in the parkinsonian SN (Spencer et al., 1998). The amino acid analysis demonstrated the presence of cysteine in NM, albeit at low levels.

Consistent with an earlier report (Carstam et al., 1991), small quantities of DA and DOPA were measured in two samples of NM isolated from two different populations and analyzed in three different laboratories. This suggests that both the DA precursor and DA itself may constitute a small proportion of the polymer. Odh et al. (1994) suggested that SN NM is a mixed pheo- and eumelanin formed from DA and cysDA and that onethird to one-half of SN NM consists of benzothiazinederived units. It is interesting that the benzothiazoles formed from cysDA are neurotoxic and have been suggested to be involved in the etiology of PD (Zhang and Dryhurst, 1994). NM production may thus be a cellular mechanism that inactivates these potentially damaging metabolites in brain regions in which the catecholamine oxidation rates are high. An interesting finding was the presence of DOPA in NM and the fact that DOPA levels are reduced following proteinase $\mathrm{K}$ treatment. Gieseg et al. (1993) have suggested that protein-bound DOPA is a major reductant formed during hydroxyl radical damage to proteins, and it is possible that such a mechanism might result in the inclusion of DOPA into NM.

The present data suggest that NM contains a proteinaceous component that forms an integral part of the polymer structure. The amino acid content we report here is also in agreement with another recent work using different isolation procedures (Zecca et al., 2000). These results, and a recent report by one of the current authors (L.Z.; Bridelli et al., 1999), of a proteinaceous component in normal NM contrast with those of Lopiano et al. (1999), who report an insoluble protein component only in NM isolated from parkinsonian brains. This difference

TABLE 2. Compounds identified in NM and DAM

\begin{tabular}{lllc}
\hline \multicolumn{1}{c}{ Sample } & DOPA & DA & $\begin{array}{c}\text { Amino acid } \\
\text { content }\end{array}$ \\
\hline DAM & 0.00 & 6.26 & ND \\
NM, no proteinase & $0.41^{a}$ & $0.32^{a}$ & $165^{a}$ \\
NM, 3-h proteinase & $0.16^{b}$ & $0.12^{b}$ & $57^{b}$ \\
\hline
\end{tabular}

Units are $\mu \mathrm{g} / \mathrm{mg}$ melanin. DOPA and DA were analyzed using $\mathrm{HI}$ hydrolysis and amino acid content by $\mathrm{HCl}$ hydrolysis as described in the text. ND, not detected.

${ }^{a} \mathrm{NM}$ isolated from Italian brains.

${ }^{b} \mathrm{NM}$ isolated from German brains. 
may result from the use of different techniques for isolating NM, although that used by Lopiano et al. (1999) is not described. The identity of this protein or these proteins was not established, but it is possible that the protein possesses an enzyme function involved in NM production. Tyrosinase, the enzyme involved in the production of peripheral melanins, has not been demonstrated in the brain (Barden, 1969; Ikemoto et al., 1998). It has been assumed that NM is formed by the nonenzymatic oxidation of DA; a dark synthetic melanin pigment can indeed be produced from DA in vitro, a process that is catalyzed by transition metals such as iron (Graham et al., 1978). Although it was believed that the pigment is formed spontaneously, more recent findings have led to the reexamination of the possibility that NM may be, at least in part, enzymatically produced.

The present results support the hypothesis that NM is a complex polymer, its components including a variety of substances involved in DA neurotransmission, as might be expected from its cellular localization in the dopaminergic neurons of the SN. We have identified some components and characteristics of this polymer, and have compared NM isolated from brain tissues of different populations. Although the present work has yielded useful insights into this most intriguing of polymers, our findings apply only to the soluble part of this substance, as under the conditions used an insoluble portion of the NM remained and could not be included in the analysis. To resolve the structure of the entire polymer, further work using solid-state NMR would be useful. The physiological role of NM and its possible involvement in the relatively selective nigral dopaminergic cell death occurring in PD remain, however elusive, and further functional investigations are required to clarify these questions.

Acknowledgment: K.L.D. was the recipient of an Alexander von Humboldt Fellowship from the Federal Republic of Germany and a C. J. Martin Research Fellowship from the National Health and Medical Research Council of Australia. The authors thank Dr. T. Arzberger and Prof. A. Weindl (Neurology Clinic, Technical University of Munich, Germany) for the provision of research tissue and R. Harth, T. Heinemann, and G. Pezzoli for technical assistance. The work was funded by the Bundesministerium für Bildung, Wissenschaft und Technologie, Germany (nos. 01KL 9013 and 01KL 9101-1) and the Deutsche Parkinson-Vereinigung e.V. P.R. and M.G. held a grant from the Deutsche Parkinson-Vereinigung e.V. M.M. and C.G. thank the Fonds der Chemischen Industrie for support. The NMR experiments were performed at the Large Scale Facility for Biomolecular NMR at the University of Frankfurt (ERB CT 9500 34). L.Z. acknowledges the support of the Italian Association for Parkinson Disease-Milano and CARIPLO Foundation-Milano.

\section{REFERENCES}

Aime S., Fasano M., Bergamasco B., Lopiano L., and Quattrocolo G. (1996) Nuclear magnetic resonance spectroscopy characterization and iron content determination of human mesencephalic neuromelanin, in Advances in Neurology (Battistini L., Scarlato G., Caraceni T., and Ruggieri S., eds), pp. 263-270. LippincottRaven Publishers, Philadelphia.
Barden H. (1969) The histochemical relationship of neuromelanin and lipofuscin. J. Neuropathol. Exp. Neurol. 28, 419-441.

Ben-Shachar D. and Youdim M. B. (1990) Selectivity of melaninized nigra-striatal dopamine neurons to degeneration in Parkinson's disease may depend on iron-melanin interaction. J. Neural Transm. Suppl. 29, 251-258.

Ben-Shachar D. and Youdim M. B. H. (1991) Intranigral iron injection induces behavioral and biochemical "parkinsonism" in rats. J. Neurochem. 57, 2133-2135.

Ben-Shachar D., Riederer P., and Youdim M. B. H. (1991) Ironmelanin interaction and lipid peroxidation: implications for Parkinson's disease. J. Neurochem. 57, 1609-1614.

Bindoli A., Rigobello M. P., and Deeble D. J. (1992) Biochemical and toxicological properties of the oxidation products of catecholamines. Free Radic. Biol. Med. 13, 391-405.

Bridelli M. G., Tampellini D., and Zecca L. (1999) The structure of neuromelanin and its iron binding site studied by infrared spectroscopy. FEBS Lett. 457, 18-22.

Carstam R., Brinck C., Hindemith-Augustsson A., Rorsman H., and Rosengren E. (1991) The neuromelanin of the human substantia nigra. Biochim. Biophys. Acta 1097, 152-160.

Crippa P. R., Wang Q. J., Eisner M., Moss S. C., Zecca L., Zschack P., and Gog T. (1996) Structure of human neuromelanin by x-ray diffraction: comparison with synthetics. Pigment Cell Res. 5, 72.

Das K. C., Abramson M. B., and Katzman R. (1978) Neuronal pigments: spectroscopic characterization of human brain melanin. J. Neurochem. 30, 601-605.

Double K. L., Riederer P. F., and Gerlach M. (1999) Significance of neuromelanin for neurodegeneration in Parkinson's disease. Drug News Dev. 12, 333-340.

Gerlach M., Ben-Shachar D., Riederer P., and Youdim M. B. H. (1994) Altered brain metabolism of iron as a cause of neurodegenerative diseases? J. Neurochem. 63, 793-807.

Gerlach M., Trautwein A. X., Zecca L., Youdim M. B. H., and Riederer P. (1995) Mössbauer spectroscopic studies of purified human neuromelanin isolated from the substantia nigra. J. Neurochem. 65, 923-926.

Gerlach M., Gsell W., Kornhuber J., Jellinger K., Krieger V., Pantucek F., Vock R., and Riederer P. (1996) A post mortem study on neurochemical markers of dopaminergic, GABA-ergic and glutamatergic neurons in basal ganglia-thalamocortical circuits in Parkinson syndrome. Brain Res. 741, 142-152.

Gibb W. R. (1992) Melanin, tyrosine hydroxylase, calbindin and substance $\mathrm{P}$ in the human midbrain and substantia nigra in relation to nigrostriatal projections and differential neuronal susceptibility in Parkinson's disease. Brain Res. 581, 283-291.

Gieseg S. P., Simpson J. A., Charlton T. S., Duncan M. W., and Dean R. T. (1993) Protein-bound 3,4-dihydroxyphenylalanine is a major reductant formed during hydroxyl radical damage to proteins. Biochemistry 32, 4780-4786.

Good P. F., Olanow C. W., and Perl D. P. (1992) Neuromelanincontaining neurons of the substantia nigra accumulate iron and aluminum in Parkinson's disease: a LAMMA study. Brain Res. 593, 343-346.

Graham D. G., Tiffany S. M., Bell W. M., and Gutknecht W. F. (1978) Autoxidation versus covalent binding of quinones as the mechanism of toxicity of dopamine, 6-hydroxydopamine, and related compounds toward C1300 neuroblastoma cells in vitro. Mol. Pharmacol. 14, 644-653.

Hirsch E., Graybiel A. M., and Agid Y. A. (1988) Melanized dopaminergic neurons are differentially susceptible to degeneration in Parkinson's disease. Nature 334, 345-348.

Ikemoto K., Nagatsu I., Ito S., King R. A., Nishimura A., and Nagatsu T. (1998) Does tyrosinase exist in neuromelanin-pigmented neurons in the human substantia nigra? Neurosci. Lett. 253, 198-200.

Ito S. and Fujita K. (1985) Microanalysis of eumelanin and pheomelanin in hair and melanomas by chemical degradation and liquid chromatography. Anal. Biochem. 144, 527-536.

Ito S., Imai Y., Jimbow K., and Fujita K. (1988) Incorporation of sulfhydryl compounds into melanins in vitro. Biochim. Biophys. Acta 964, 1-7. 
Jellinger K., Kienzl E., Rumpelmair G., Riederer P., Stachelberger H., Ben-Shachar D., and Youdim M. B. H. (1992) Iron-melanin complex in substantia nigra of parkinsonian brains: an x-ray microanalysis. J. Neurochem. 59, 1168-1171.

Langston J. W., Forno L. S., Tetrud J., Reeves A. G., Kaplan J. A., and Karluk D. (1999) Evidence of active nerve cell degeneration in the substantia nigra of humans years after 1-methyl-4-phenyl-1,2,3,6tetrahydropyridine exposure. Ann. Neurol. 46, 598-605.

Li H. and Dryhurst G. (1997) Irreversible inhibition of mitochondrial complex I by 7-(2-aminoethyl)-3,4-dihydro-5-hydroxy- $2 \mathrm{H}-1,4-$ benzothiazine-3-carboxylic acid (DHBT-1): a putative nigral endotoxin of relevance to Parkinson's disease. J. Neurochem. 69, $1530-1541$.

Lopiano L., Digilio G., Fasano M., Giraudo S., Rizzone M., Torre E., and Bergamasco B. (1999) Iron and neuromelanin in Parkinson's disease. J. Neural Transm. 106, XXIV.

Odh G., Carstam R., Paulson J., Wittbjer A., Rosengren E., and Rorsman H. (1994) Neuromelanin of the human substantia nigra: a mixed-type melanin. J. Neurochem. 62, 2030-2036.

Peter M. G. and Förster H. (1989) On the structure of eumelanins: identification of constitutional patterns by solid-state NMR spectroscopy. Angew. Chem. Int. Ed. Engl. 28, 741-743.

Pilas B., Sarna T., Kalyanaraman B., and Swartz H. M. (1988) The effect of melanin on iron associated decomposition of hydrogen peroxide. Free Radic. Biol. Med. 4, 285-293.

Rosengren E., Linder-Eliasson E., and Carlsson A. (1985) Detection of 5-S-cysteinyldopamine in human brain. J. Neural Transm. 63, 247-253.

Sarna T., Menon I. A., and Sealy R. C. (1985) Photosensitization of melanins: a comparative study. Photochem. Photobiol. 42, 529532.

Sarna T., Pilas B., Land E. J., and Truscott T. G. (1986) Interaction of radicals from water radiolysis with melanins. Biochim. Biophys. Acta 883, 162-167.

Shen X. M. and Dryhurst G. (1998) Iron- and manganese-catalyzed autoxidation of dopamine in the presence of L-cysteine: possible insights into iron- and manganese-mediated dopaminergic neurotoxicity. Chem. Res. Toxicol. 11, 824-837.
Shen X. M., Zhang F., and Dryhurst G. (1997) Oxidation of dopamine in the presence of cysteine: characterization of new toxic products. Chem. Res. Toxicol. 10, 147-155.

Smythies J. (1996) On the function of neuromelanin. Proc. R. Soc. Lond. [Biol.] 263, 487-489.

Spencer J. P. E., Jenner P., Daniel S. E., Lees A. J., Marsden D. C., and Halliwell B. (1998) Conjugates of catecholamines with cysteine and GSH in Parkinson's disease: possible mechanisms of formation involving reactive oxygen species. J. Neurochem. 71, 2112-2122.

Wakamatsu K., Ito S., and Nagatsu T. (1991) Cysteinyldopamine is not incorporated into neuromelanin. Neurosci. Lett. 131, 57-60.

Youdim M. B., Ben-Shachar D., and Riederer P. (1989) Is Parkinson's disease a progressive siderosis of substantia nigra resulting in iron and melanin induced neurodegeneration? Acta Neurol. Scand. 126, 47-54.

Zareba M., Bober A., Korytowski W., Zecca L., and Sarna T. (1995) The effect of a synthetic neuromelanin on yield of free hydroxyl radicals generated in model systems. Biochim. Biophys. Acta 1271, 343-348.

Zecca L. and Swartz H. M. (1993) Total and paramagnetic metals in human substantia nigra and its neuromelanin. J. Neural Transm. Park. Dis. Dement. Sect. 5, 203-213.

Zecca L., Mecacci C., Seraglia R., and Parati E. (1992) The chemical characterization of melanin contained in substantia nigra of human brain. Biochim. Biophys. Acta 1138, 6-10.

Zecca L., Pietra R., Goj C., Mecacci C., Radice D., and Sabbioni E. (1994) Iron and other metals in neuromelanin, substantia nigra, and putamen of human brain. J. Neurochem. 62, 1097-1101.

Zecca L., Shima T., Stroppolo A., Goj C., Battiston G. A., Gerbasi R., Sarna T., and Swartz H. M. (1996) Interaction of neuromelanin and iron in substantia nigra and other areas of human brain. Neuroscience 73, 407-415.

Zecca L., Costi P., Mecacci C., Ito S., Terreni M., and Sonnino S. (2000) The interaction of human substantia nigra neuromelanin with lipids and peptides. J. Neurochem. 74, 1758-1765.

Zhang F. and Dryhurst G. (1994) Effects of L-cysteine on the oxidation chemistry of dopamine: new reaction pathways of potential relevance to idiopathic Parkinson's disease. J. Med. Chem. 37, 1084-1098. 